Supporting Information

\title{
Kinetics of Coupled Primary- and Secondary-Minimum Deposition of Colloids under Unfavorable Chemical Conditions
}

CHONGYANG SHEN, BAOGUO LI, YUANFANG HUANG, AND YAN JIN

Number of pages: 3

Number of figures: 1

Number of tables: 1 


\section{Supporting Information}
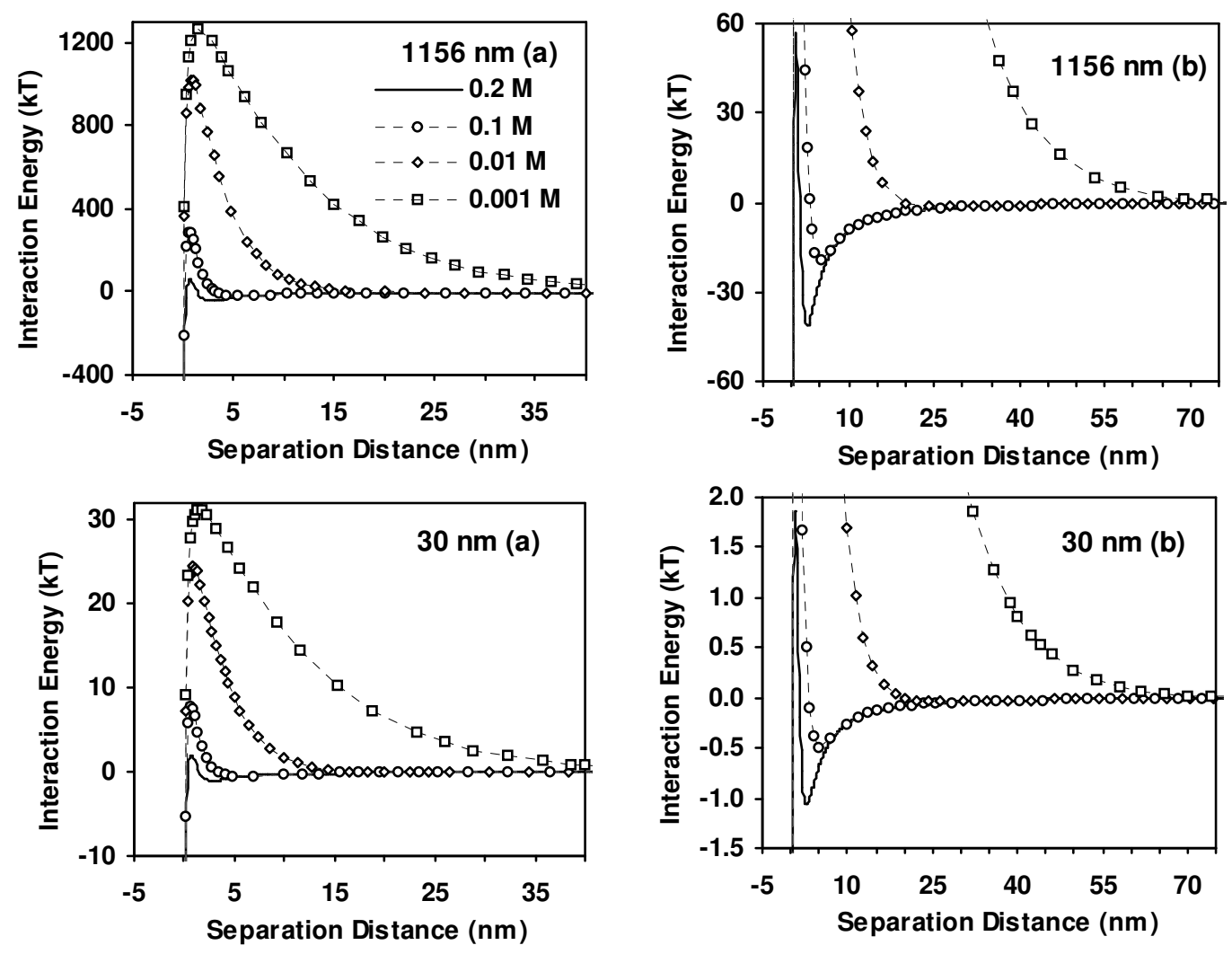

Fig.S1 DLVO energy profiles for the $1156 \mathrm{~nm}$ and $30 \mathrm{~nm}$ colloids $\left(A=1 \times 10^{-20} \mathrm{~J}, T=298\right.$ K). (a) Calculated interaction energy as a function of separation distance at different ionic strengths; (b) Re-plotted profiles to highlight the depth and location of secondary minimum.

Table S1. Zeta potentials of sand and colloids (1156 nm and $30 \mathrm{~nm})$, and calculated maximum energy barriers $\left(\Phi_{\max }\right)$, secondary-minimum $\left(\Phi_{\mathrm{sec}}\right)$ depths and distances for both colloids at different ionic strengths.

\begin{tabular}{cccccccccc}
\hline$I$ & \multicolumn{3}{c}{ Zeta Potential $(\mathrm{mV})$} & \multicolumn{2}{c}{$\Phi_{\max }(k T)$} & \multicolumn{4}{c}{$\Phi_{\text {sec }}$} \\
$(\mathrm{M})$ & Septh $(k T)$ & \multicolumn{2}{c}{ Distance $(\mathrm{nm})$} \\
& Sand & $\mathbf{1 1 5 6} \mathbf{~ n m}$ & $30 \mathbf{n m}$ & $\mathbf{1 1 5 6} \mathbf{~ n m}$ & $30 \mathbf{n m}$ & $\mathbf{1 1 5 6} \mathbf{~ n m}$ & $30 \mathbf{n m}$ & $\mathbf{1 1 5 6} \mathbf{~ n m}$ & $30 \mathbf{n m}$ \\
\hline 0.001 & -39.27 & -46.73 & -44.3 & 1267.70 & 31.31 & 0.11 & 0.003 & 110.13 & 109.46 \\
0.01 & -39.13 & -46.46 & -43.25 & 1019.47 & 24.41 & 1.54 & 0.04 & 26.03 & 25.71 \\
0.1 & -32.08 & -38.04 & -38.69 & 291.80 & 7.83 & 19.60 & 0.51 & 5.12 & 5.15 \\
0.2 & -27.49 & -35.21 & -36.86 & 59.94 & 1.97 & 41.30 & 1.05 & 2.81 & 2.87 \\
\hline
\end{tabular}




\section{Maxwell Model}

The velocity distribution of the colloids in the secondary minimum follows the function:

$$
f(v)=4 \pi\left(\frac{m_{p}}{2 \pi k T}\right)^{(3 / 2)} v^{2} \exp \left(-\frac{m_{p} v^{2}}{2 k T}\right)
$$

where

$$
\int_{0}^{\infty} f(v) d v=1
$$

and $m_{p}$ is mass of a colloid and $v$ is its velocity. Here a dimensionless kinetic energy of the colloid is defined as

$$
x^{2}=\frac{m_{p} v^{2}}{2 k T}
$$

Then equation (1) simplifies to

$$
f(v)=4 x^{2} \exp \left(-x^{2}\right) \sqrt{\frac{m_{p}}{2 \pi k T}}
$$

According to assumption (ii), the fraction of successful collisions (arrival at the separation distance corresponding to the secondary minimum) that result in deposition in the secondary minimum, $\alpha_{\text {sec }}$, is given by

$$
\alpha_{\mathrm{sec}}=\int_{0}^{v_{\mathrm{sec}}} f(v) d v=\int_{0}^{v_{\mathrm{sec}}} 4 x^{2} \exp \left(-x^{2}\right) \sqrt{\frac{m_{p}}{2 \pi k T}} d v=\int_{0}^{\sqrt{\Phi_{\mathrm{sec}}}} \frac{4}{\pi^{1 / 2}} x^{2} \exp \left(-x^{2}\right) d x
$$

where $\Phi_{\mathrm{sec}}=m v_{\text {sec }}{ }^{2} / 2 k T$. Similarly, the fraction of successful collisions resulting in colloid deposition in the primary minimum, $\alpha_{p r i}$, is

$$
\alpha_{p r i}=\int_{\sqrt{\Delta \Phi}}^{\infty} \frac{4}{\pi^{1 / 2}} x^{2} \exp \left(-x^{2}\right) d x
$$

The total fraction of successful collisions $\alpha$ (sum of $\alpha_{s e c}$ and $\alpha_{p r i}$ ) is written as

$$
\alpha=\alpha_{p r i}+\alpha_{\mathrm{sec}}=1-\int_{\sqrt{\Phi_{\mathrm{sec}}}}^{\sqrt{\Delta \Phi}} \frac{4}{\pi^{1 / 2}} x^{2} \exp \left(-x^{2}\right) d x
$$

